\title{
Genomic Evidences Support an Independent History of Grapevine Domestication in the Levant
}

\author{
Aviad Sivan ${ }^{1}$, Oshrit Rahimi², Mail Salmon-Divon ${ }^{3}$, Ehud Weiss $^{4}$, Elyashiv Drori ${ }^{2,5^{*}}$, Sariel \\ Hübner $^{*}$ \\ ${ }^{1}$ Galilee Research Institute (Migal), Tel-Hai Academic College, Upper Galilee 12210, Israel \\ ${ }^{2}$ Department of Chemical engineering, Ariel University, Ariel 40700, Israel \\ ${ }^{3}$ Department of Molecular Biology, Ariel University, Ariel, Israel \\ ${ }^{4}$ Department of Land of Israel Studies and Archaeology, Bar Ilan University, Israel \\ ${ }^{5}$ Eastern regional R\&D Center, Ariel 40700, Israel. \\ *These authors contributed equally
}

\begin{abstract}
The ancient grapevines of the Levant have inspired beliefs and rituals in human societies which are still practiced today in religious and traditional ceremonies around the world. Despite their importance, the original Levantine wine-grapes varieties were lost due to cultural turnovers commencing in the $7^{\text {th }}$ century $\mathrm{CE}$, which lead to the collapse of a flourishing winemaking industry in this region. Recently, a comprehensive survey of feral grapevines was conducted in Israel in an attempt to identify local varieties, yet the origin of these domesticated accessions is unclear. Here we study the origin of Levantine grapevines using whole-genome sequence data generated for a diversity panel of wild and cultivated accessions. Comparison between Levantine and Eurasian grapevines indicated that the Levantine varieties represent a distinct lineage from the Eurasian varieties. Demographic models further supported this observation designating that domestication in the Caucasus region predated the emergence of the Levantine samples in circa 5000 years and that authentic descendants of ancient varieties are represented among the Levantine samples. We further explore the pedigree relationship among cultivated grapevines, identify footprints of selective sweeps, and estimate the extent of genetic load in each group. We conclude that the Levantine varieties are distinct from the Eurasian varieties and that resistance to disease and abiotic stress are key traits in the development of both Eurasian and Levantine varieties.
\end{abstract}

Keywords: grapevine, domestication, demography, transposable elements, whole-genome sequencing, selective sweep 


\section{Introduction}

Since ancient times, domesticated grapevine (Vitis vinifera ssp. sativa) had an inspiring role in the culture of human societies. Among grapevine products, wine is the most popular and influential, thus many legends and believes were tied with its consumption in the Mediterranean region and the Near East $(1,2)$. Today, the grapevine is among the most valuable horticulture crops in the world, cultivated on over 7 million ha. around the globe, mostly for wine production (3).

According to archaeological evidences, the grapevine was domesticated 8,000-10,000 years ago in the Taurus, Caucasus and Zagros Mountains (4). However, molecular evidences from chloroplast DNA suggest at least one more domestication event outside the Near East (5) albeit not conclusively (6). From the Caucasian region, domesticated grapevines have presumably distributed southwards to the Levant and later to Europe $(1,4)$. Tracing the history of grapevines is challenging due to its spread as vegetative propagation material and mixtures between different genetic sources $(2,7)$. Thus, the history of grapevine domestication and distribution remains largely obscure (8).

Recent genomic evidences allowed to study the domestication history of grapevine with higher confidence and to date the split between domesticated European varieties from wild grapevines to circa $22 \mathrm{~K}$ years ago $(9,10)$. These estimates significantly predate archaeological evidences, presumably due to a missing link to the direct wild ancestral population. Thus, the exact originating population of the domesticated grapevine is still a missing key in the domestication history of cultivated grapevines.

In the Levant region, winemaking was highly abundant at least since the Bronze age and has contributed significantly to the culture and economy of ancient societies. This important ancient industry has flourished for many centuries until the collapse of the Byzantine empire in the region during the $7^{\text {th }}$ century CE (11). For five centuries the wine industry was suppressed but continued to exist until the conquest of the Mamluk empire in the $13^{\text {th }}$ century when winemaking, cultivation, or production became completely forbidden $(12,13)$. Consequently, Levantine wine grapevine varieties which had an important role in ancient culture in this region for many centuries were abandoned and considered lost thereafter. Recently, a comprehensive grapevine survey was conducted in an attempt to revive the ancient Levantine wine industry (14). Based on a panel of SSR markers, the Levantine populations were clustered in a separate clade from European varieties (15). However, it is unclear whether the collected varieties are authentic ancient Levantine varieties or rather the outcome of a more recent introduction of European varieties during the $19^{\text {th }}$ century. 
Here, we present the analysis of whole-genome sequencing data obtained for 81 domesticated (sativa) and wild (sylvestris) accessions representing a diversity panel of Levantine and Eurasian grapevines. We provide new evidences for the domestication history of grapevines in the Levant which support the authenticity of this material. Our results indicate that at least a few of the grapevine varieties that were cultivated in the Levant in ancient times survived the suppression of the wine industry in the region commencing in the $7^{\text {th }}$ century. In addition, genomic screening of the different populations provided evidences that the Levantine and Eurasian sativa lineages are distinguishable and that selective sweeps have affected known domestication syndrome traits in both groups in addition to group-specific loci.

\section{Results}

Population stratification in Eurasian and Levantine grapevines. A panel of 81 grapevine accessions was obtained from two independent sources. The Levantine panel included 46 putative domesticated sativa types and 9 wild sylvestris accessions (Fig. 1A) that were selected from a recently established collection genotyped previously with 22 SSR markers (15). Selected accessions were confirmed to be unique material based on the SSR markers and were whole-genome sequenced (WGS) to a coverage of 20X. In addition, publicly available raw WGS data for 17 Eurasian sativa varieties and 9 Eurasian sylvestris accessions were obtained from the SRA repository (9). Altogether 2.1 trillion bp were obtained for 81 grapevine accessions representing domesticated sativa and wild sylvestris types of Levantine and Eurasian origin. High quality trimmed reads from all accessions were aligned to the Pinot Noir PN40024 reference genome (16) followed by a variant calling procedure, yielding a total of 26,083,120 high quality (QUAL > 20) SNPs across all accessions. To further reduce the false positive rate among called variants, a machine-learning filtering approach was implemented and a set of 1,824,029 robustly called SNPs were kept for downstream analyses.

To investigate the population stratification among Levantine and Eurasian accessions, modelbased analyses were conducted using fastStructure (17) (Fig. 1B, Fig. S1) and ADMIXTURE (Fig. S2) (18). Considering both analyses, the optimal clustering was obtained at $\mathrm{K}=4$ in accordance with the geographic origin and type of accessions: Levantine sativa, Levantine sylvestris, Eurasian sativa, and Eurasian sylvestris. Signs of admixture were observed among all four groups with the highest rate of admixed individuals in the Eurasian sylvestris group (100\%), and lowest admixture rate in the Levantine sylvestris (33\%). The difference in the level of admixture observed among the two sylvestris groups conceivably reflects the 
distribution range of each group, i.e. broad and narrow geographic range represented in the Eurasian and Levantine groups, respectively.

Overall, the assignment of accessions to clusters well supported previous characterization records with minor exceptions. Two accessions in the Eurasia sativa group ('ThompsonRLK', 'Thompson2A') known as table-grapes varieties were assigned to a distinct cluster from the remaining wine-grapes type. The same pattern was also observed in three Levantine sativa accessions ('Tamar-H1', 'Tamar-H2', and 'Suka') which were assigned to the same cluster as the two 'Thompson' varieties based on the fastStructure analysis. These results support previous reports for both Eurasian (9) and Levantine accessions (15). Strong signs of admixture were observed in specific accessions from both the Eurasian and Levantine sativa groups. For example, among the Levantine sativa accessions, a high rate of admixture with Levantine sylvestris (65\%) was observed for the 'Buffalo' accession, presumably due to recent hybridization with wild Levantine grapevine (Fig.1 B). Among the Eurasian sativa accessions, high rates of admixture were observed in 'Zinfandel'/'Primitivo' (56\%), 'Muscat of Alexandria' and 'Carignan' (74\%).

To further investigate the level of divergence between the four grapevine groups, a neighborjoining network was build using the SNP dataset called across all 81 accessions (Fig. 1C). The split into four groups in the network analysis supported the results of the model-based stratification analyses. Moreover, the obtained network clearly discriminated between the Eurasian and Levantine groups, implying that the Eurasian sativa group has branched from the Eurasian sylvestris group while the Levantine sativa group has branched from the Levantine sylvestris group. This pattern of divergence suggests that the Eurasian and Levantine sativa lineages do not share the same domestication history and may have developed in independent processes. To further confirm this observation, a PCA was conducted and designated the same pattern of divergence as obtained in the neighbor-joining network and model-based stratification analyses (Fig. S3). In addition, the network analysis well supported the mis-assignment of accessions identified by the model-based stratification analyses. Interestingly, one wild white-berry accession collected near the Sea of Galilee ('Majrase') was confirmed as a sylvestris type by all analyses. White-berry is a common phenotype among domesticated grapevines and considered a post-domestication characteristic (19). A white-berry phenotype in wild grapevine is possibly the result of introgression from cultivated sativa, however, none of the analyses conducted supported 
signs of introgression from cultivated grapevines into this wild white-berry accession.

Next, the level of nucleotide diversity $(\pi)$, Watterson's $\theta$, Tajima's $D$, observed heterozygosity, and linkage disequilibrium (LD) were investigated in each group after excluding five mis-assigned accessions (defined as less than 10\% assignment to their expected cluster). Linkage disequilibrium analysis conducted across all Vitis accessions indicated that decay is reached at $20 \mathrm{Kbp}$ (Fig. S4, Table S1). Within groups, steep LD decay and high genetic diversity were observed among the Eurasian Sylvestris ( $\pi=0.29 \pm 0.01$, LD $=8 \mathrm{Kbp})$ compared with the Eurasian sativa group $(\pi=0.27 \pm 0.01, \mathrm{LD}=10 \mathrm{Kbp})$ which is characterized by a significantly lower diversity $\left(t=3.50, p=1.20 \times 10^{-4}\right)$ due to domestication bottleneck (Table S1). An opposing trend was observed among the Levantine groups, i.e. steep LD decay and high genetic diversity within the Levantine sativa group $(\pi=0.27 \pm 0.01$, $\mathrm{LD}=6 \mathrm{Kbp})$ compared with Levantine Sylvestris $(\pi=0.25 \pm 0.01, \mathrm{LD}=25 \mathrm{Kbp})$ which was also characterized by a significantly lower diversity $\left(t=6.35, p=2.30 \times 10^{-7}\right)$. This pattern among the Levantine groups is attributed to the constrained geographic range of wild grapevines at its southern distribution edge (15). In addition, both the population stratification and genetic diversity analyses imply that the Levantine sativa group is likely a mixture of two distinct subgroups (Fig 1B). Thus, the domesticated Levantine sativa group was further split into two subgroups (Table S1), a homogenous Levantine sativa, and a highly diverse group with signs of admixture with other genetic sources.

The demographic history of Eurasian and Levantine grapevines. To test the hypothesis that Eurasian and Levantine sativa are distinct lineages that were developed in two independent processes, various complementary demographic analyses were conducted. To reduce the confounding effect of differences in sample size and level of admixture across groups, nine representative accessions were selected from each group to adjust the sample size to the smallest groups (nine accessions in Eurasia sylvestris and Levantine sylvestris). To explore historical splits and gene-flow among the four groups, a graphical model-based analysis was conducted using TreeMix (20). The SNP dataset was restricted to intergenic regions to reduce the effect of selective sweeps on the demographic inferences, thus a total of $1,055,512$ SNPs was kept for the analysis. The model uses the allele frequencies in modern populations and a Gaussian approximation to infer historical demographic events. First, the model was carried out without migration $\left(f_{\text {index }}=0.996\right)$ and the obtained graph supported the hypothesis of independent demographic history (Fig. 2A). Although the model does not allow 
to precisely estimate times of split events, it was noted that domestication in Eurasia predated the development of the Levantine sativa. Allowing one migration event in the model improved its likelihood $\left(f_{\text {index }}=0.999\right)$ and indicated a historical gene-flow from Levantine sativa into the Eurasia sativa group (Fig. 2B). This inference supports previous reports on the possible exchange of germplasm by the Romans, crusaders, or Islamic rulers (2). Allowing more migration events in the model did not improve its likelihood (Fig. S5).

To further investigate the demographic history of grapevines in Eurasia and the Levant, a coalescence analysis was conducted using the $\mathrm{SMC}++$ program (21) which allows to incorporate information from all nine individuals representing each group. To infer the demographic changes on a timescale, a generation time of three years and a mutation rate of $5.4 \times 10^{-9}$ per nucleotide per generation were used in the model (Fig. $2 \mathrm{C}$ ).

The SMC++ analysis was conducted and inspected with and without the 'clean-split' model to allow a better interpretation of the demographic process. In all models, the divergence between groups predated the 'clean-split' model indicating that grapevine domestication was prolonged over a period of time until the two groups were fixed as distinct lineages. In agreement with previous studies $(9,10)$, the $\mathrm{SMC}++$ model denoted that the divergence between Eurasian sylvestris and sativa grapevines commenced circa 30,000 years ago and reached a clear differentiation about 15,000 years ago based on the 'clean-split' model (Fig. 2C). Conducting the analysis for the Levantine sativa group indicated that divergence from Eurasian sylvestris and sativa predates the estimated domestication event in Eurasia $(20,000$ and 17,000 years ago, respectively). The same demographic analysis between Levantine sylvestris and sativa indicated that divergence between these groups commenced approximately 15,000 years ago but they reached a clear differentiation 10,000 years ago according to the 'clean-split' model. Considering the small distribution range represented among Levantine sylvestris accessions and the long period of divergence, it is unlikely that the wild population represented in this study is the direct progenitor of Levantine sativa. Nevertheless, the demographic inferences for the Levantine sativa group provide evidence that these accessions are authentic Levantine varieties that sustained the suppression of grapevines cultivation and winemaking industry in the Levant. Arguably, the descendants of the lost Levantine wine varieties that were cultivated in this region in ancient times.

To further validate these results, a second analysis was conducted with the MSMC software (22) using four individuals that were randomly sampled from each population. The obtained trajectories in the effective population size of each group over time supported the observed split between the Eurasian sylvestris and sativa grapevines circa 20,000 years ago and a more 
recent split between the Levantine sylvestris and sativa grapevines circa 9,000 years ago. The MSMC inferences accord with the results obtained by the SMC++ and TreeMix analyses (Fig. S6).

\section{Pedigree network in domesticated Eurasian and Levantine varieties. To better}

understand the recent history and pedigree relationship among grapevine varieties, a relatedness network was constructed for all 58 domesticated (sativa) accessions of both Levantine and Eurasian origin. The relatedness matrix was computed at $1 \mathrm{cM}$ segments using the refined-IBD tool (23) which allows to identify traces of shared ancestry signatures. This approach has the advantage of distinguishing between 'old' ancestry (short segments) and recent ancestry (long segments) signatures. The minimum threshold to delineate a parentoffspring or sibling relationship was determined based on a confirmed cutoff $(\mathrm{IBD}=0.466$ ) which was computed using a short segments ancestry detection approach (7). To link between this confirmed cutoff and the calculated refined-IBD score, a correlation analysis was conducted between overlapping pairs of accessions present in both studies (spearman: rho = $0.62, p=0.028$ ). To graphically visualize the pedigree network, a first-degree relationship graph was constructed using Gephi (24) which allows organizing accessions in an attractionrepulsion network (Fig. 3). Overall, the Levantine sativa formed a distinguished cluster from the Eurasian sativa group which further support the independent history of the Levantine domesticated accessions. Clonal relationships were identified in both the Eurasian ('Zinfandel'/‘Primitivo') and Levantine ('Zeni-A'/'Suriman-S'/'Asba-el-arus') groups. Interestingly, a pedigree relationship was observed between the European 'Chardonnay' variety and Levantine accessions sampled at the Galilee ('Margaliot-1', 'Hadari').

Footprints of domestication in cultivated verities. To investigate whether the independent history of the Levantine and Eurasia sativa has resulted in distinct patterns of selective sweeps we performed a genome scan analysis using the $\mu$-statistic score (25) that was calculated for the 9 representative accessions from each group. The $\mu$-statistic is a composite score combining the site frequency spectrum (SFS), linkage disequilibrium, and genetic diversity calculated at overlapping windows that are adaptively determined according to the calculated metrics. We considered a significant outlier window following the default cutoff of 99.95\% (Fig. 4). Altogether, 5,581 outlier windows were detected in the Eurasia sativa group and 6,333 outlier windows were detected in the Levantine sativa. The average $\mu$ statistic in the Levantine sativa group was significantly higher than in the Eurasian sativa 
group $(t=10.6, p<0.0001)$. The overall stronger signal of selective sweep detected in the Levantine sativa group may indicate that this group has experienced stronger selection or that it was developed more recently. To identify footprints of a selective sweep as a result of domestication and breeding, overlapping windows were merged and extended to form quantitative trait regions (QTRs). Merging was performed only for outlier windows found in distance shorter than $20 \mathrm{Kbp}$ in accordance with LD decay in vitis. A total of 222 and 260 outlier QTRs were detected across all chromosomes in the Eurasian and Levantine sativa groups, respectively (Table S2). Among the identified QTRs, 80 were found in both groups across all chromosomes except for chromosome 8 where no QTR was detected in neither group. Enrichment in the number of QTRs was observed in the Eurasian sativa group on chromosomes 12 (26 QTRs), 13 (17 QTRs), and 19 (22 QTRs), and a strong signal of selection $(\mu>100)$ was observed on chromosomes 7 (103.1) and 13 (145.5). In the Levantine sativa group, enrichment in the number of QTRs was observed on chromosomes 4 (25 QTRs), 5 (32 QTRs), and 18 (21 QTRs), and a strong signal of selection ( $\mu>100)$ was observed on chromosomes 2 (110.8), 11 (751.5), 12 (106), 17 (409.8), and 18 (150.4).

Several candidate genes were found within outlier QTRs in both Eurasian and Levantine sativa. For example, the genes resveratrol synthase and stilbene synthase that are involved in the response to biotic stress as well as in taste and aroma (26) were found in an overlapping QTR on chromosome 16 in both groups. Another example was observed on chromosome 2 in both sativa groups where bifunctional nitrilase/nitrile hydratase genes were found. These genes were recently targeted for their potential role in grapevine domestication $(9,27)$. Among the Levantine sativa group, a strong signal of a selective sweep was observed on chromosome 18 where a cluster of phenylalanine ammonia-lyase (PAL) genes was identified. PAL genes contribute to anthocyanin concentration in the berry pericarp which affects berry color and wine quality in addition to enhancing resistance to biotic and abiotic stresses (28). In the same QTR located on chromosome 18, we also detected an RPW8 gene that confers a basal resistance to powdery mildew in Arabidopsis (29). Other QTRs that were detected exclusively in the Levantine sativa group were on chromosome 17 where several abiotic stress-responsive genes were identified including the basic helix-loop-helix transcription factor (30) and HVA22-like gene (31,32).

Outlier QTRs that were detected exclusively in the Eurasian sativa group harbored the anthocyanin synthesis genes (MYBA1 and MYBA3) on chromosome 2 $(32,33)$, the pathogen response gene (ACD6) on chromosome 16 (35), and the disease response genes (RPM1) on 
chromosome 7 which were described to be up-regulated in response to pathogen infection in grapevine (36).

Genetic load among cultivated grapevines. To evaluate the extent of genetic load due to the accumulation of deleterious mutations along the genome in each of the sativa groups we used the SIFT4G software (37). A total of 92,936 non-synonymous variants were detected of which 37,635 were predicted as deleterious (SIFT score $<0.05$ ). To correct for potential bias introduced by the use of a reference genome, alleles identified also in the outgroup species $V$. rotundifolia were not considered deleterious (9). Altogether 29,386 sites were variants were identified as deleterious across all accessions and used to calculate the genetic load in each group. Not surprisingly, the calculated genetic load in the Levantine sativa group $(7,142 \pm$ 101) was significantly higher $\left(t=4.51 p=1.70 \times 10^{-4}\right)$ than in the Levantine sylvestris group $(6,228 \pm 598)$ due to the effect of domestication. Moreover, significantly higher genetic load $\left(t=14.70, p=1.24 \times 10^{-4}\right)$ was observed in the Levantine sativa group compared with the Eurasian sativa $(5,168 \pm 390)$. This observation supports the results obtained in the selective sweep analysis indicating that the Levantine group was under stronger selective pressure or was generated more recently.

\section{Discussion}

Along the history of human societies, wine has provided a special cultural flavor to the life of ancient and modern societies. According to archaeological observations, the grapevine was domesticated and spread by ancient societies in the Near East (circa 10,000 BC), and later was introduced to East Asia, the Mediterranean basin and Europe $(4,10)$.

In the Levant region, grapevine cultivation has flourished for several millennia until the collapse of the Byzantine empire during the $7^{\text {th }}$ century CE (11). Since then, wine production has declined and eventually abandoned under the Mamluk empire conquest, and the ancient grapevine varieties were considered lost $(12,13)$. In this study, we provide new genomic evidences for the demographic history of grapevine varieties in the Levant, their origin, and the genomic landscape of their domestication.

The origin of cultivated grapevines in the Levant. To study the history of Levantine varieties we compared whole-genome sequence data from sylvestris and sativa types of Levantine and Eurasian origin. All population stratification analyses supported the deviation 
into four distinct groups by type (sylvestris/sativa), and geography (Levant/Eurasia).

Moreover, the clustering pattern implied that the Eurasian sativa group has branched from the Eurasian sylvestris group while the Levantine sativa group branched from the Levantine sylvestris group, with few minor exceptions (Fig. 1). In accordance with previous studies (9), the Eurasian table-grapes varieties ('Thompson') and 'Muscat of Alexandria' are distinguished from remaining varieties and a similar pattern was also observed among three Levantine sativa accessions which presumably represent introduced table-grapes varieties ('Tamar-H1', 'Tamar-H2', 'Suka'). Also, one Levantine accession was identified as a potential feral grapevine ('Buffalo'), and one white-berry accession ('Majrase') was confirmed to be of wild origin. The latest could be an interesting example of a sporadic occurrence of white-berry mutation in the wild, as we failed to identify signs of admixture with other domesticated varieties.

The process of domestication can be generally divided into three phases which include management of wild material, selection of desirable basic domestication traits, and dispersal of the domesticated material. During the third stage of domestication, introgression from local wild populations can increase adaptation of the alien crop to the local environment $(8,38)$. These introgressions may be spread across the genome yet the genetic background should reflect the origin of the crop. Once the genetic turnover is so profound that the origin is masked by introgression, the question of the origin of domesticates becomes quantitative. The demographic analyses conducted in this study supported an independent domestication history of the Levantine sativa since no traces of late introgressions were identified. However, we cannot exclude based on our analyses a complete genomic turnover of the original domesticated material in the Near East. In accordance with previous studies $(9,10)$, our analyses pointed that the divergence of Eurasian sativa from sylvestris commenced approximately 30 thousand years ago, however, the 'clean-split' model in SMC++ provided an improved estimate for the domestication of grapevine in Eurasia to approximately 15,000 years ago. It should be noted that these models are limited in their ability to estimate recent demographic events especially in the past few centuries (21). Therefore, it is difficult to infer robustly from these models whether the Levantine accessions are truly descendants of ancient varieties or the outcome of a recent introduction of grapevine varieties from Eurasia. The pedigree and genome scan analyses imply these are indeed authentic Levantine varieties.

Pedigree relationship between Eurasian and Levantine varieties. To test for potential recent admixture between Levantine and Eurasian sativa, and the effect of vegetative 
propagation on the similarity between cultivars (7), a relatedness network was constructed based on pair-wise identity by descent (IBD) analysis. In accordance with previous studies (7) conducted for the Eurasia sativa, clonal relationship was observed among the Eurasian accessions and also among the Levantine accessions but no pedigree links were observed between the two groups with one exception. A pedigree link between the European 'Chardonnay' variety and Levantine accessions sampled at the Galilee region ('Margaliot-1', 'Hadari') imply a potential Levantine ancestry for 'Chardonnay'. Previously, ampelographers suggested that 'Chardonnay' has ancestral roots in the Levant, although it was later contradicted by genetic analysis which indicated that 'Chardonnay' was produced by a cross between 'Pinot Noir' and 'Gouais blanc' $(39,40)$. While 'Pinot Noir' is a confirmed French variety, 'Gouais blanc' is considered an introduced variety from elsewhere. The results obtained from the pedigree analysis do not allow to track back the entire lineage of 'Chardonnay' and how it is linked to the Levant, but to the best of our knowledge, this is the first genomic evidence for this ancestry relationship.

\section{Development of grapevine varieties in Eurasia and the Levant.}

All population stratification, demography, and pedigree analyses conducted supported the hypothesis that Levantine sativa originated from a different source than the Eurasian sativa. To test whether these independent histories are reflected in different genomic footprints of domestication, genome scans were conducted for each sativa group. Footprints of selective sweeps were detected across all chromosomes in both groups with many overlapping QTRs. However, several group-specific QTRs were also detected and included candidate genes that are involved in biotic and abiotic stress resistance. Environmental stress has always troubled farmers and breeders around the world, however, since the pathogen identity and type of stress varies between regions, so are the resistance genes selected to confront them.

The revolution of genomics provides a powerful tool to fill gaps in the history of crops at multiple genomic levels (41). Despite their importance in human culture and economy, many ancient Levantine grapevines varieties were consider lost for many centuries. Here we provided evidences that the Levantine ancient grapevine varieties survived, in some cases under harsh conditions, despite the cultural turnovers in this region along history.

Nevertheless, to link between the discovered Levantine accessions and ancient varieties we need to obtain high-quality sequence data from archaeological samples which is now in reach also for grapevines. 


\section{Materials and methods}

Whole-genome sequencing and variant calling: Samples were obtained from two separate sources (Table S3). Levantine samples were selected from a recently established large Vitis collection comprised of 372 accessions which were genotyped using 22 standard SSR markers (15). Based on this data, clone accessions were removed and a representative diversity panel of 55 accessions was selected for our study including 46 accessions identified as sativa and 9 accessions identified as sylvestris. From each accession, a young leaf tissue (shoot tip) was sampled for DNA extraction using the QIAmp DNA Micro cleanup kit (Qiagen, Valencia, USA), and a library was prepared using the NEBNext Ultra DNA library preparation kit (catalog number E7370L; NEB). Whole-genome sequencing of 150bp pairedend reads was generated on Illumina HiSeq2500 machine to a target coverage of 20X per sample. Raw sequence data for 17 Eurasian sativa and 9 sylvestris samples were obtained from the short-read archive (SRA) project number PRJNA388292 (9). Altogether, wholegenome sequence data of 81 accessions were obtained and analyzed.

The quality of raw sequence data for all Levantine and Eurasian samples was inspected using FastQC v0.11.8 (42). Low-quality reads were trimmed and adapters were removed using Trimmomatic v.0.32 (43) with default parameters. Following trimming, reads were inspected again with FastQC to guarantee only high-quality reads are included in the analysis. Clean reads from each sample were aligned to the Pinot Noir 40024 reference genome (16) using BWA-MEM (44) with default parameters. The obtained alignment files were further processed following the GATK best practice protocol (45) and included marking duplicates with picard-tools v.2.8.1 (46), realignment around indels with GATK (47) and indexing using samtools v.1.3.1 (48). Variant calling was conducted across all 81 accessions in one batch using the HaplotypeCaller program and variants were filtered using the variant quality score recalibration (VQSR) algorithm as implemented in GATK v3.6 (47). Briefly, the VQSR uses a machine-learning algorithm to develop a model of true variants based on validated SNPs and allows the discrimination between true and false calls. We used the 20K Illumina SNPchip data (49) as a training set in the VQSR analysis and a minimum LOD score of 4 was set to guarantee that the highest confident SNPs are kept for downstream analyses. The obtained SNP set was further filtered to exclude sites with more than $20 \%$ missing data across all samples and a minimum minor allele frequency of $5 \%$. 
Population stratification analyses. For population structure analysis we used both the FastStructure v1.0 (17) and ADMIXURE v1.3.0 (18) programs. For each analysis, the number of ancestral populations $(\mathrm{K})$ tested ranged from 2 to 10 with 20 replicates for each $\mathrm{K}$. The cross-validation procedure implemented in ADMIXTURE and the 'chooseK' tool implemented in FastStructure were used to select the most likely number of clusters explaining the population structure among vitis accessions.

A neighbor-joining (NJ) network was constructed in SpitsTrees4 (50) using all SNPs that passed the filtering procedure. In addition, a principal component analysis (PCA) was conducted using the smartPCA program as implemented in EIGNSOFT v6.1.4 package (51).

Demographic analyses. To infer the historical relationship including events of splits and migrations among populations, we used the TreeMix v1.13 program (20). To avoid sample size bias on demographic inferences the number of accessions in each group was set to nine individuals in accordance with the smallest groups (sylvestris groups). In each sativa group, the 9 accessions with the highest ancestry assignment, as obtained by FastStructure, were selected for downstream analyses. To avoid bias introduced by genomic regions affected by non-neutral processes, the SNP dataset was restricted to intergenic regions. In addition, SNPs were called in a Vitis rotundifolia accession (Muscadine, SRA accession number: SRR5627788) (9) which was used in the model as an outgroup for rooting the tree. To filter non-independent SNPs in the model, windows were set to the size of twice the calculated average number of SNPs per 20Kbp (the evaluated extent of LD across populations).

Additionally, zero to four migration events were tested in the model.

To further infer the demographic history and split time in the Levantine and Eurasian populations, we used the likelihood-free $\mathrm{SMC}++$ program v1.15.2(21). The $\mathrm{SMC}++$ program allows to leverage information from multiple individuals of each population and infer changes in the effective population size also in recent history. A mutation rate of $\mu=5.4 \times 10^{-9}$ (10) mutations per base-pair per generation, and a generation time of 3 years were assumed in all models. The input data from each population was filtered for long stretches of homozygosity ( $>20 \mathrm{Kbp}$ ) and the cross-validation (CV) module was used to infer the effective population sizes with 10 -fold CV steps. A polarization error rate was set to 0.5 to allow uncertainty on the identity of the ancestral allele. Finally, the 'clean-split' model was used with default parameters to estimate split times between pairs of populations. 
In addition, the MSMC v2 program (22) was used to estimate changes in the effective population size $\left(N_{\mathrm{e}}\right)$ in each population over time. Four individuals were randomly chosen from each population after phasing and imputing the entire SNP data set using BEAGLE v5.1 (39). A mutation rate of $\mu=5.4 \times 10^{-9}$ mutations per base-pair per generation and a generation time of three years were set in the model.

Pedigree network analysis. To explore the breeding history of grapevine verities in the Levant and their relationship to the Eurasian varieties, a pair-wise identity by descent (IBD) was calculated across all domesticated sativa accessions (Eurasia and Levant). The RefinedIBD program in BEAGLE v5.1 (23) was used to calculate the pair-wise IBD, and the results were converted to a kinship score using the 'relatedness' tool as implemented in BEAGLE v5.1 (39). A relatedness threshold of 0.466 was set following a previous study (7) on the familial relationship among grapevine varieties based on IBD scores calculated in the program plink (52). To adjust the relatedness scores calculated from the Refined-IBD to the pre-calculated threshold, a Spearman correlation analysis was conducted between the Refined-IBD and the plink scores computed for each pair of accessions. The obtained pairwise IBD matrix was visualized with the network analysis program Gephi v0.9.2 (24) using the multi-gravity force-atlas 2 algorithm. This algorithm allows to transpose the IBD kinship in the attraction-repulsion 2D network and the sub-networks were identified using the modularity algorithm which considers both quality and quantity of the pair-wise links.

Population genomics statistics. Linkage disequilibrium (LD) was computed using the PopLDdecay software (53) across all accessions and for each population separately. LD was also measured within each population at $1 \mathrm{Mbp}$ windows using plink (52). Population diversity statistics were calculated for each group separately using the PopGenome package (54) and included nucleotide diversity $(\pi)$, Tajima's D, and Watterson's $\theta$. Observed heterozygosity was obtained for each population using VCFtools v0.1.15 (55).

Genome scans for the footprint of selective sweeps were conducted within each domesticated sativa group. Within each group, the $\mu$-statistic was calculated using the RAiSD software for 9 accessions from each group (25). The $\mu$-statistic is a composite score of the changes in site frequency spectrum (SFS), linkage disequilibrium, and genetic diversity. Top-ranked windows (>99.95\%) were considered outliers. Overlapping windows 
with a maximum gap of 20Kbp were merged with BEDtools v2.26.0 (56) to allow a comparison of genomic regions between groups.

Genetic load estimation. To estimate the genetic load in each accession we first identified non-synonymous mutations using the Vitis vinifera reference genome (NCBI_Assembly: GCF_000003745.3) and associated annotation files (annotations release 102, GCF_000003745.3_12X) to build a SIFT genomic database in SIFT4G (37). The identified non-synonymous mutations within coding regions were considered as potentially deleterious if the obtained SIFT score was lower than 0.05 . To avoid a reference bias effect on the genetic load predictions, alleles identified also in the outgroup species Vitis rotundifolia were not considered deleterious. The genetic load was calculated by summing the number of deleterious alleles in each accession with a score of one for heterozygote and two for homozygote deleterious alleles.

\section{Acknowledgment:}

This work was supported by the JNF grant \# 90-23-020-12, and the general support of the Israeli Ministry of Science and Technology (MOST).

Author contribution: ED, EW, and SH designed this study; AS performed the research; AS, OR and ED analyzed the data; and AS, ED, EW, and SH wrote the manuscript. 


\section{References}

1. D. Zohary, M. Hopf, and E. Weiss, Domestication of plants in the Old World. $4^{\text {th }}$ Revised edition. Oxford University Press, Oxford (2012).

2. P. This, T. Lacombe, M. R. Thomas, Historical origins and genetic diversity of wine grapes. Trends Genet. 22, 511-519 (2006).

3. Food and agriculture organization of the united nations. http://www.fao.org/faostat.

4. P. E. McGovern, Ancient wine: The search for the origins of viniculture. Anc. Wine Search Orig. Vinic. 58, 1-365 (2013).

5. R. Arroyo-García, et al., Multiple origins of cultivated grapevine (Vitis vinifera L. ssp. sativa) based on chloroplast DNA polymorphisms. Mol. Ecol. 15, 3707-3714 (2006).

6. M. K. Aradhya, et al., Genetic structure and differentiation in cultivated grape, Vitis vinifera L. Genet. Res. 81, 179-192 (2003).

7. S. Myles, et al., Genetic structure and domestication history of the grape. Proc. Natl. Acad. Sci. U. S. A. 108, 3530-3535 (2011).

8. Y. Zhou, A. Muyle, B. S. Gaut, Evolutionary genomics and the domestication of grapes. The Grape Genome, D. Cantu, M. A. Walker, Eds. Springer International Publishing, pp. 39-55 (2019).

9. Y. Zhou, M. Massonnet, J. S. Sanjak, D. Cantu, B. S. Gaut, Evolutionary genomics of grape (Vitis vinifera ssp. vinifera) domestication. Proc. Natl. Acad. Sci. U. S. A. 114, 11715-11720 (2017).

10. Z. Liang, et al., Whole-genome resequencing of 472 Vitis accessions for grapevine diversity and demographic history analyses. Nat. Commun. 10, 1-12 (2019).

11. R. Frankel, Presses for oil and wine in the southern Levant in the Byzantine period. Dumbart. Oaks Pap. 51, 73-84 (1997).

12. A. Levanoni, Food and cooking during the Mamluk era: Social and political implications. Annales 23, 1017-53 (1968). Mam. Stu. Rev. 9, 222-201 (2005).

13. Z. Safrai, and A. M. Maier. The rural landscape of ancient Israel. 114 (2003).

14. E. Drori, et al., Ampelographic and genetic characterization of an initial Israeli grapevine germplasm collection. J. Gra. Res. 54, 107-110 (2015).

15. E. Drori, et al., Collection and characterization of grapevine genetic resources (Vitis vinifera) in the Holy Land, towards the renewal of ancient winemaking practices. Sci. Rep. 7 (2017).

16. O. Jaillon, et al., The grapevine genome sequence suggests ancestral hexaploidization in major angiosperm phyla. Nature 449, 463-467 (2007). 
17. A. Raj, M. Stephens, J. K. Pritchard, FastSTRUCTURE: Variational inference of population structure in large SNP data sets. Genetics 197, 573-589 (2014).

18. D. H. Alexander, J. Novembre, K. Lange, Fast model-based estimation of ancestry in unrelated individuals. Genome Res. 19, 1655-1664 (2009).

19. Z. Migicovsky, et al., Patterns of genomic and phenomic diversity in wine and table grapes. Hortic. Res. 4, 1-11 (2017).

20. J. K. Pickrell, J. K. Pritchard, Inference of population splits and mixtures from genome-wide allele frequency data. PLoS Genet. 8 (2012).

21. J. Terhorst, J. A. Kamm, Y. S. Song, Robust and scalable inference of population history from hundreds of unphased whole genomes. Nat. Genet. 49, 303-309 (2017).

22. S. Schiffels, R. Durbin, Inferring human population size and separation history from multiple genome sequences. Nat. Genet. 46, 919-925 (2014).

23. A. L. Browning, S. R. Browning, Improving the accuracy and efficiency of identity-bydescent detection in population data. Genetics 194, 459-471 (2013).

24. M. Bastian, S. Heymann, M. Jacomy, Gephi: An open source software for exploring and manipulating networks. Int. AAAI Conf. Weblogs Soc. Media, 361-362 (2009).

25. N. Alachiotis, P. Pavlidis, RAiSD detects positive selection based on multiple signatures of a selective sweep and SNP vectors. Commun. Biol. 1, 1-11 (2018).

26. A. Walker, D. Cantau, The grape genome. Springer International Publishing (2019).

27. A. Marrano, D. Micheletti, S. Lorenzi, D. Neale, M. S. Grando, Genomic signatures of different adaptations to environmental stimuli between wild and cultivated Vitis vinifera $\mathrm{L}$. Hortic. Res. 5, 1-12 (2018).

28. K. A. Roubelakis-Angelakis, W. M. Kliewer, Effects of exogenous factors on phenylalanine ammonia-lyase activity and accumulation of anthocyanins and total phenolics in grape berries. Am. J. Enol. Vitic. 37, 275-280 (1986).

29. S. Xiao, et al., The atypical resistance gene, RPW8, recruits components of basal defense for powdery mildew resistance in Arabidopsis. Plant J. 42, 95-110 (2005).

30. M. Gao, et al., Identification of the grape basic helix-loop-helix transcription factor family and characterization of expression patterns in response to different stresses. Plant Growth Regul. 88, 19-39 (2019).

31. Q. Shen, C. N. Chen, A. Brands, S. M. Pan, T. H. David Ho, The stress- and abscisic acid-induced barley gene HVA22: Developmental regulation and homologues in diverse organisms. Plant Mol. Biol. 45, 327-340 (2001). 
32. B. N. Chen, C. C. Chu, R. Zentella, S. M. Pan, T. H. D. Ho, AtHVA22 gene family in Arabidopsis: Phylogenetic relationship, ABA and stress regulation, and tissue-specific expression. Plant Mol. Biol. 49, 633-644 (2002).

33. A. M. Fischer, et al., Quantitative trait locus analysis of fungal disease resistance factors on a molecular map of grapevine. Theor. Appl. Genet. 108, 501-515 (2004).

34. M. Salmaso, et al., A grapevine (Vitis vinifera L.) genetic map integrating the position of 139 expressed genes. Theor. Appl. Genet. 116, 1129-1143 (2008).

35. Z. Zhang, J. Shrestha, C. Tateda, J. T. Greenberg, Salicylic acid signaling controls the maturation and localization of the arabidopsis defense protein ACCELERATED CELL DEATH6. Mol. Plant 7, 1365-1383 (2014).

36. W. Zhang, et al., Transcriptional response of grapevine to infection with the fungal pathogen Lasiodiplodia theobromae. Sci. Rep. 9, 1-12 (2019).

37. R. Vaser, S. Adusumalli, S. N. Leng, M. Sikic, P. C. Ng, SIFT missense predictions for genomes. Nat. Protoc. 11, 1-9 (2016).

38. M. D. Purugganan, Evolutionary insights into the nature of plant domestication. Curr. Biol. 29, 705-714 (2019).

39. J. Bowers, et al., Historical genetics: The parentage of Chardonnay, Gamay, and other wine grapes of northeastern France. Science 285, 1562-1565 (1999).

40. H. V. Hunt, M. C. Lawes, M. A. Bower, J. W. Haeger, C. J. Howe, A banned variety was the mother of several major wine grapes. Biol. Lett. 6, 367-369 (2010).

41. Y. Zhou, et al., The population genetics of structural variants in grapevine domestication. Nat. Plants 5, 965-979 (2019).

42. A. Simon., FastQC: a quality control tool for high throughput sequence data. Available at: https://www.bioinformatics.babraham.ac.uk/projects/fastqc/ (2010).

43. A. M. Bolger, M. Lohse, B. Usadel, Trimmomatic: A flexible trimmer for Illumina sequence data. Bioinformatics 30, 2114-2120 (2014).

44. H. Li, R. Durbin, Fast and accurate long-read alignment with Burrows-Wheeler transform. Bioinformatics 26, 589-595 (2010).

45. S. Schmidt, The Genome Analysis Toolkit. Proc. Int. Conf. Intellect. Capital, Knowl. Manag. Organ. Learn. 20, 254-260 (2009).

46. Picard Toolkit. Broad Institute, GitHub Repos. Available at: https://github.com/broadinstitute/picard (2019).

47. R. Poplin, et al., Scaling accurate genetic variant discovery to tens of thousands of samples. bioRxiv, 201178 (2017). 
48. H. Li, et al., The Sequence Alignment/Map format and SAMtools. Bioinformatics 25, 2078-2079 (2009).

49. M-C. Le Paslier, et al. The GRAPERESEQ 18K Vitis genotyping chip. In IX Int. Symp. Grap. Phys. Biot.. 2013.

50. C. H. Huson, D. Bryant, Application of phylogenetic networks in evolutionary studies. Mol. Biol. Evol. 23, 254-267 (2006).

51. A. L. Price, et al., Principal components analysis corrects for stratification in genome-wide association studies. Nat. Genet. 38, 904-909 (2006).

52. S. Purcell, et al., PLINK: A tool set for whole-genome association and populationbased linkage analyses. Am. J. Hum. Genet. 81, 559-575 (2007).

53. C. Zhang, et al. PopLDdecay: a fast and effective tool for linkage disequilibrium decay analysis based on variant call format files. Bioinformatics 35, 1786-1788 (2019).

29. A. Pfeifer, U. Wittelsbürger, S. E. Ramos-Onsins, M. J. Lercher, PopGenome: An efficient swiss army knife for population genomic analyses in R. Mol. Biol. Evol. 31, 1929-1936 (2014).

30. P. Danecek, et al., The variant call format and VCFtools. Bioinformatics 27, 21562158 (2011).

31. A. R. Quinlan, I. M. Hall, BEDTools: A flexible suite of utilities for comparing genomic features. Bioinformatics 26, 841-842 (2010). 
bioRxiv preprint doi: https://doi.org/10.1101/2020.07.11.198358; this version posted July 12, 2020. The copyright holder for this preprint (which was not certified by peer review) is the author/funder, who has granted bioRxiv a license to display the preprint in perpetuity. It is made available under aCC-BY-NC-ND 4.0 International license.
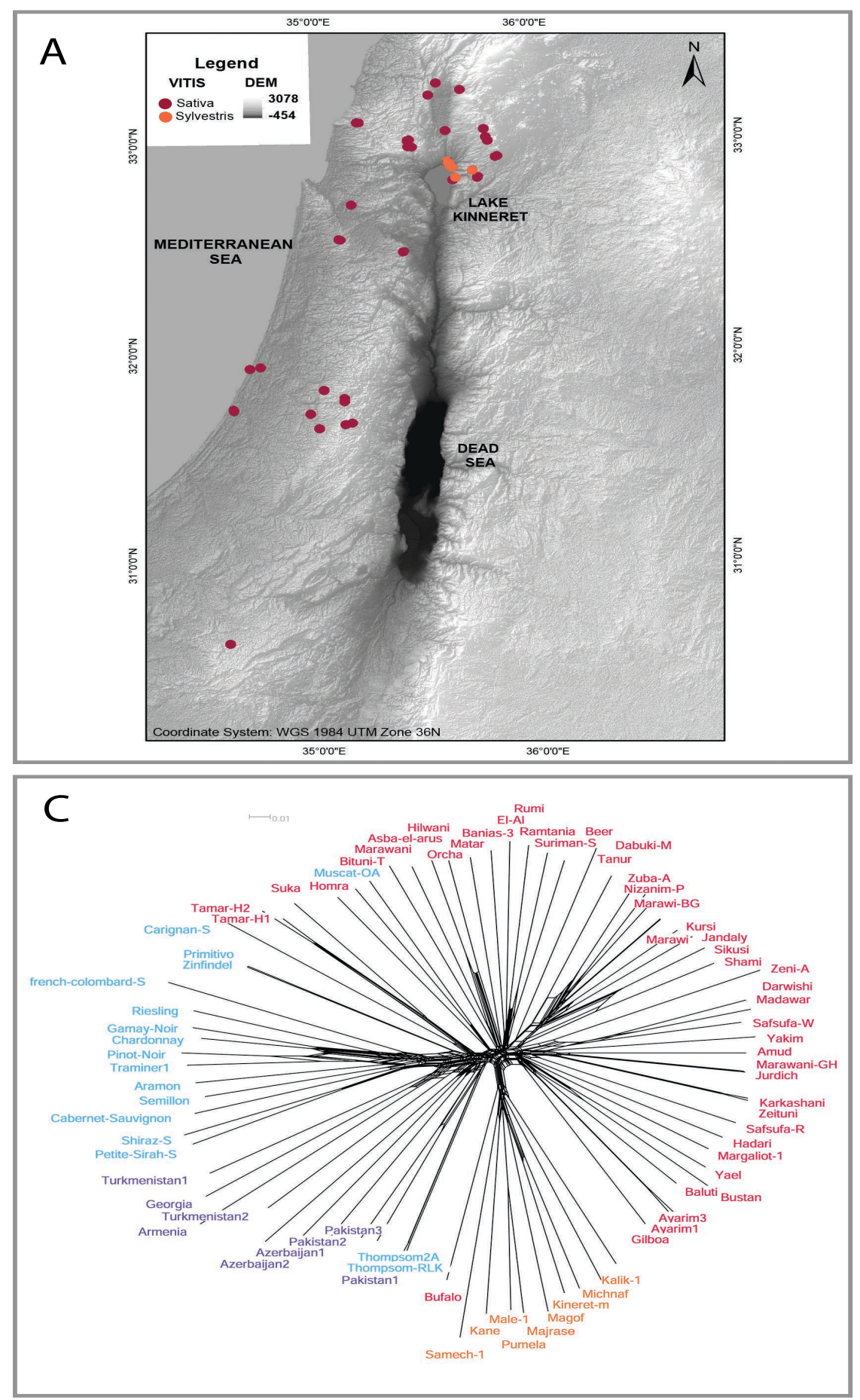

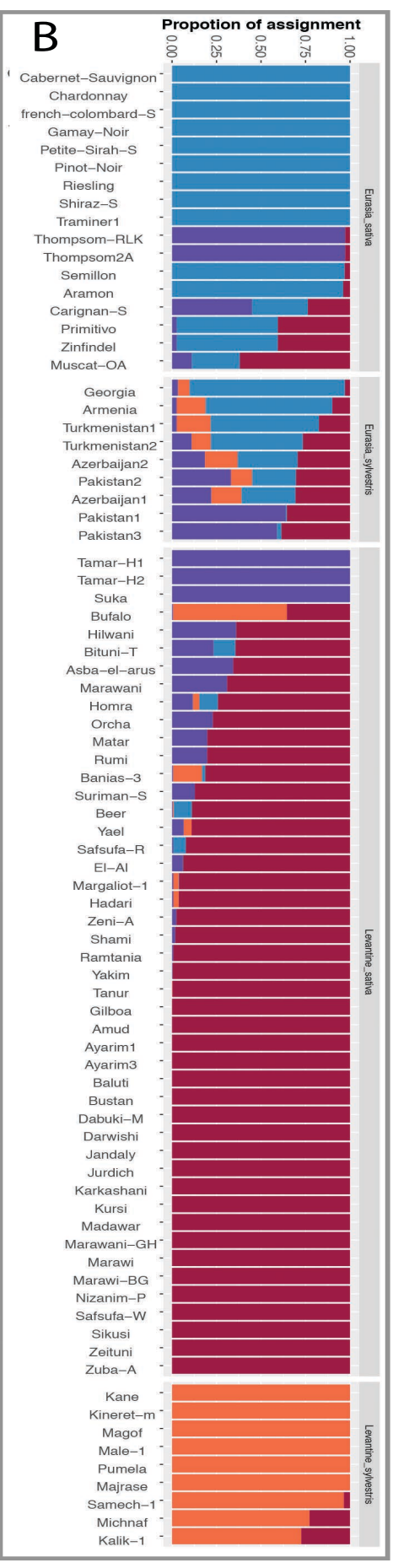

Figure1. Population structure among all Eurasian and Levantine domesticated sativa and wild sylvestris grapevine accessions. A) Geographic map and locations where Levantine sativa (orange) and sy/vestris (green) accessions were collected. B) Population structure among the 81 Eurasian and Levantine accessions. Analysis was conducted in FastSTRUCTURE and the barplot represents $K=4$. Accessions are sorted by their expected group (top-bottom): domesticated Eurasian sativa, wild Euroasian sylvestris, domesticated Levantine sativa and wild Levantine sylvestris. C) Neighbor-joining network representing the resemblance between Eurasian sativa (red), Eurasia sy/vestris (purple), Levantine sativa, and Levantine sylvestris (celeste) accessions. 

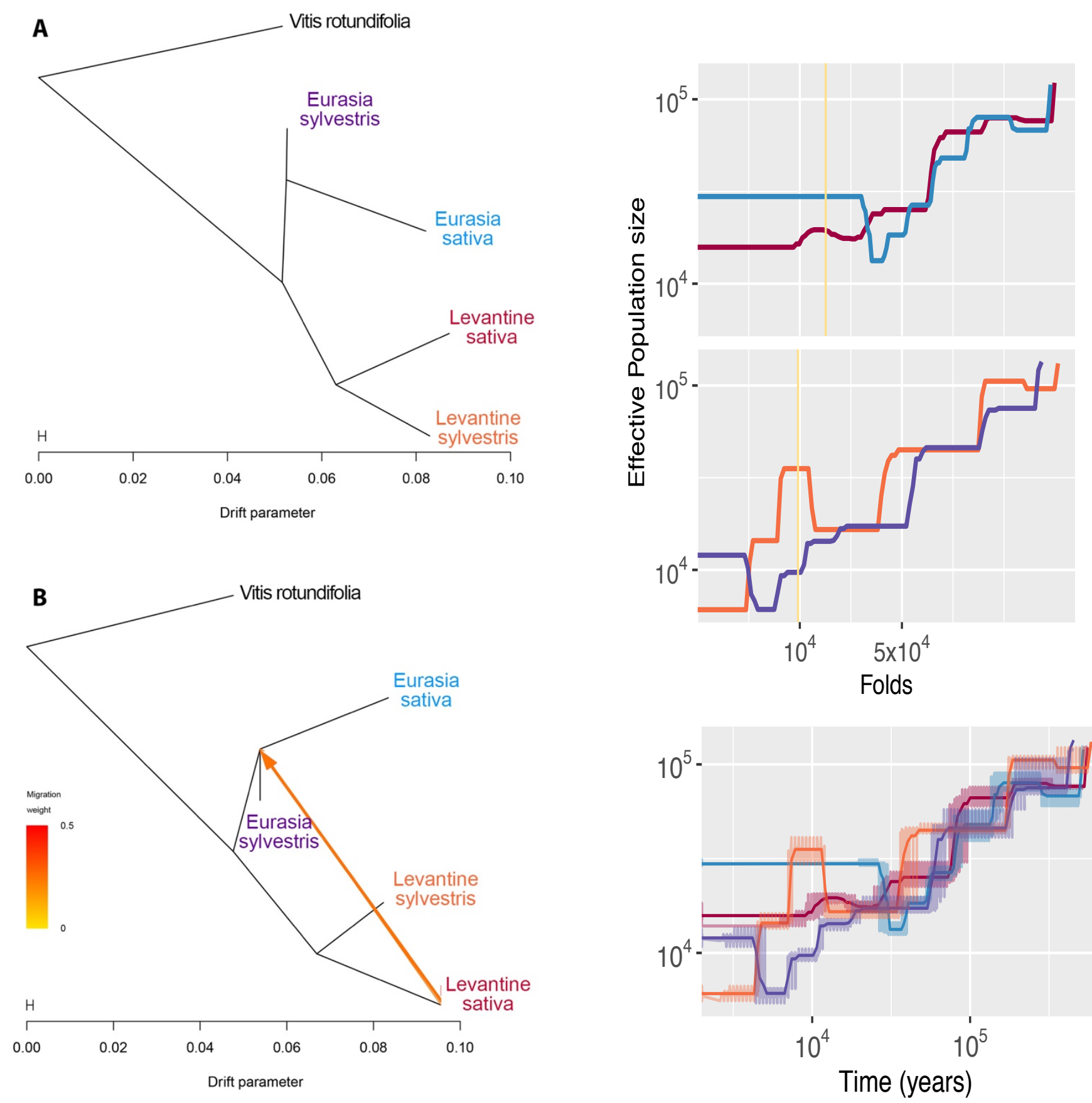

Figure2. Demographic analysis of nine representative accessions of each of the four grapevine groups: Eurasian sativa (blue), Eurasian sylvestris (purple), Levantine sativa (red) and Levantine sy/vestris (orange). A+B) Inference of splits and gene-flow among the four grapevine groups. The analysis was conducted in TreeMix using Vitis rotundifolia as an outgroup to root the tree. Presented are the results for the analysis without migration $(A)$ and with one migration event (B). When migration was allowed in the model, direction of the migration is indicated with arrow and its color represent the migration weight. C) Demographic history inference among the four grapevine groups. Analysis was conducted with SMC++ for all four groups without the 'clean split' model (bottom) and for the Eurasian sylvestris-sativa pair and Levantine sylvestris-sativa pair with the 'clean split' model (top). The estimated split time in the models is indicated with yellow vertical line. The $x$-axes represent time in years where the leftmost part correspond to recent time. The $y$-axes correspond the estimated effective population size. Each SMC++ analysis was conducted with 10 cross-validations procedures and 20 iterations. Confidence intervals are indicated with light colors in the plot. 


\section{Zeni-A Asba-el-arus \\ Suriman-S}

\section{Zinfindel \\ Primitivo}

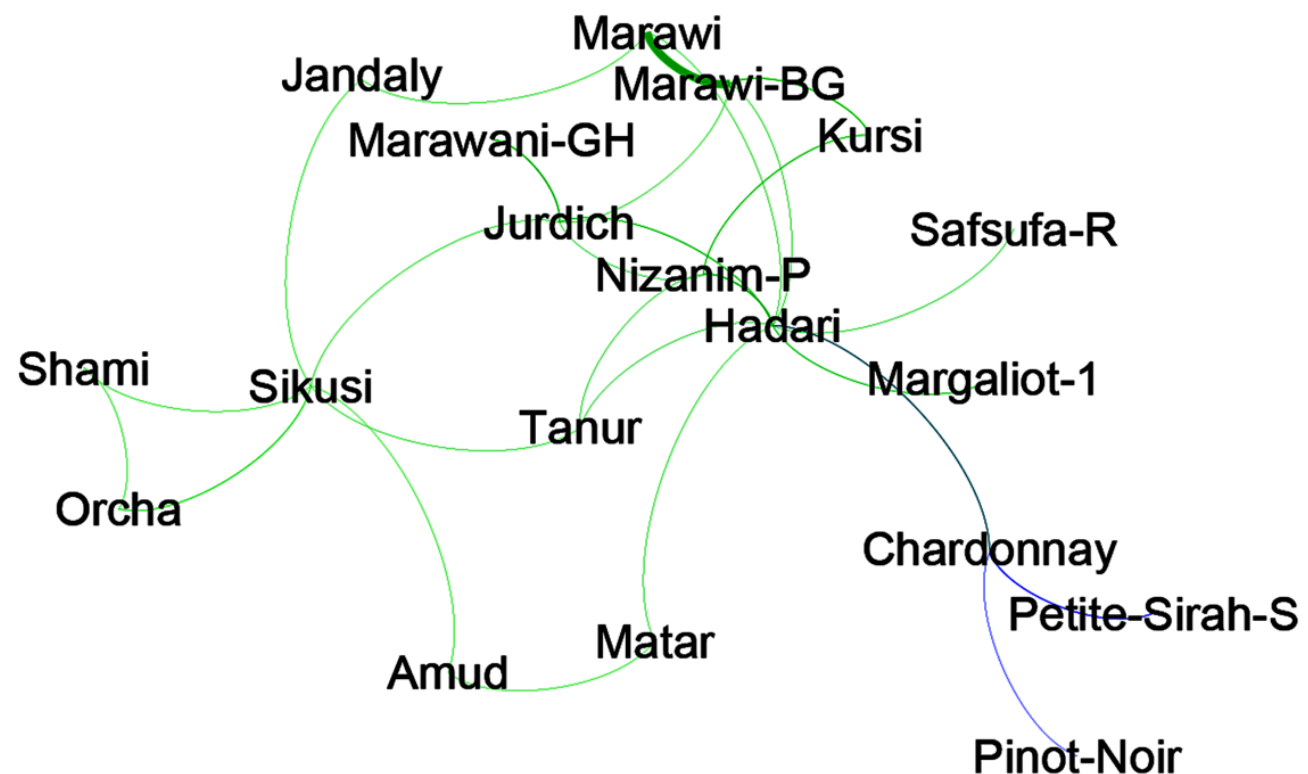

Figure3. Relatedness among domesticated Eurasian and Levantine sativa accessions. Relatedness was calculated from identity by descent (IBD) in 1cM fragments across all 57 sativa accessions. In the network, only strong links higher than 0.466 are indicated and the thickness of the line represents the link strength. Links between Levantine accessions is indicated with green edges and links between Eurasian accessions are indicated in blue edges. 
bioRxiv preprint doi: https://doi.org/10.1101/2020.07.11.198358; this version posted July 12, 2020. The copyright holder for this preprint (which was not certified by peer review) is the author/funder, who has granted bioRxiv a license to display the preprint in perpetuity. It is made available under aCC-BY-NC-ND 4.0 International license.
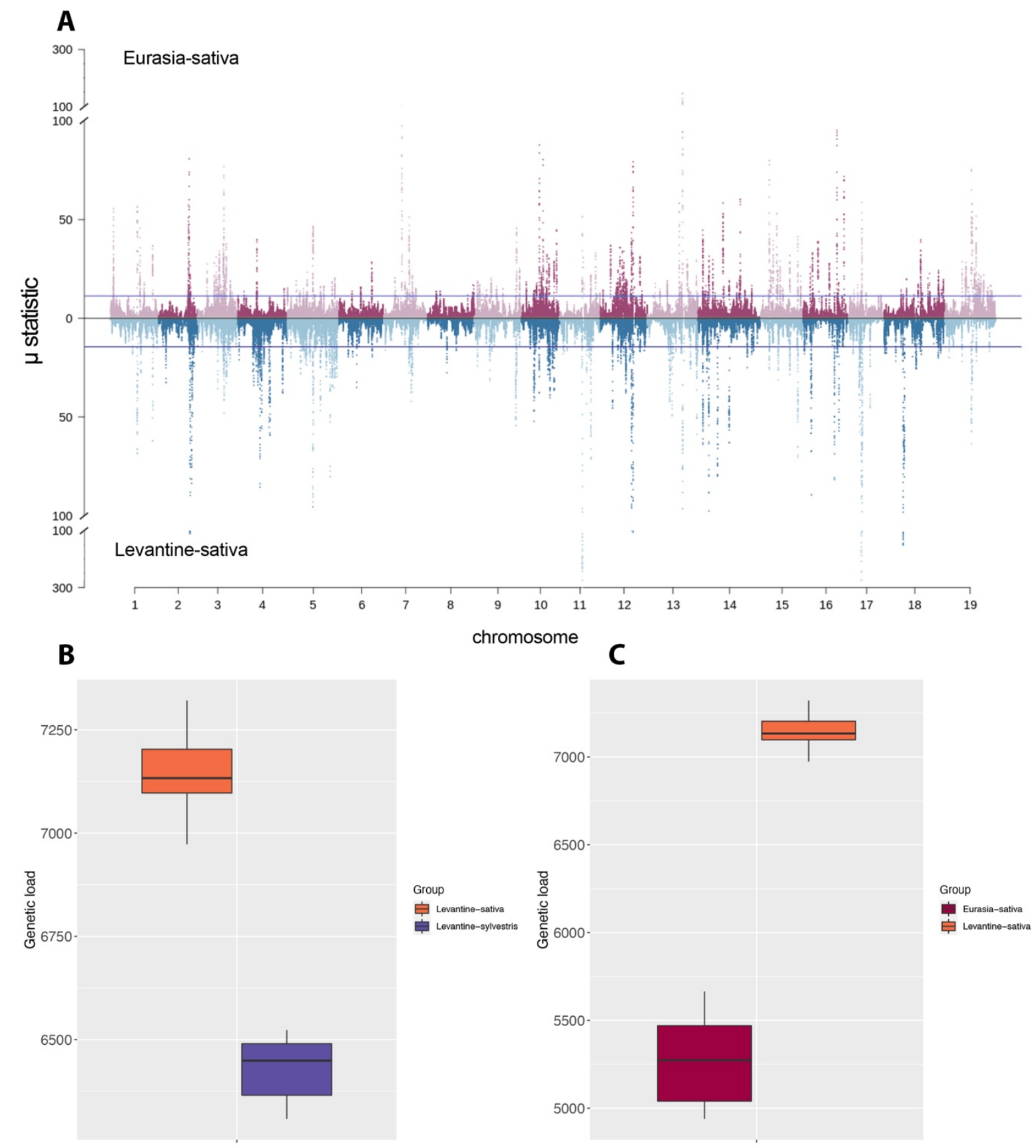

Figure 4. A) Signatures of selective sweeps in Levantine and Eurasian grapevines. Selection signal was measured using the $\mu$-statistic in sliding windows. Each dot represents a SNP score in the Levantine sativa (blue) and Eurasian sativa (red). B) Comparison between genetic load calculated in Levantine sativa and Levantine sylvestris groups. C) Comparison between genetic load calculated in Levantine sativa and Eurasian sativa groups. 